\title{
Mise à jour sur la santé mentale positive chez les adultes au Canada
}

\author{
Mélanie Varin, M. Sc.; Elia Palladino, B. Sc. S.; Tanya Lary, M.A.; Melissa Baker, Ph. D.
}

Diffuser cet article sur Twitter

\section{Résumé}

Cet aperçu présente la dernière mise à jour des estimations du Cadre d'indicateurs de surveillance de la santé mentale positive pour les adultes de 18 ans et plus. À l'aide des données de l'Enquête sur la santé dans les collectivités canadiennes de 2015 et de 2017, nous avons calculé la prévalence d'une santé mentale positive et de ses déterminants connexes. Les estimations des résultats en matière de santé mentale positive pour les adultes variaient entre $68,1 \%$ et $87,1 \%$. Nous avons aussi exploré les associations entre divers facteurs sociodémographiques et une santé mentale positive chez les adultes au Canada. Nos résultats font état de différences sociodémographiques dans les cotes portant sur la santé mentale autoévaluée, le bonheur, la satisfaction à l'égard de la vie, le bien-être psychologique et le bien-être social.

Mots-clés : santé mentale positive, adulte, santé publique, Canada

\section{Introduction}

Selon l'Agence de la santé publique du Canada (ASPC), la santé mentale est « la capacité qu'a chacun d'entre nous de ressentir, de penser et d'agir de manière à améliorer son aptitude à jouir de la vie et à relever les défis auxquels il est confronté. Il s'agit d'un sentiment positif de bien-être émotionnel et spirituel qui tient compte de l'importance de la culture, de l'équité, de la justice sociale, des interactions et de la dignité personnelle $»^{1}$. Cette définition est similaire à d'autres définitions de la santé mentale reconnues à l'échelle internationale ${ }^{2}$. L'ASPC reconnaît que la promotion de la santé mentale est essentielle à la santé et au bien-être, et l'a ciblée comme une priorité clé 3 . De ce fait, la santé mentale positive (SMP) et le bienêtre au Canada doivent être surveillés de façon continue, afin que l'on dispose d'un portrait à jour de la situation.

En 2016, l'ASPC a élaboré le Cadre d'indicateurs de surveillance de la santé mentale positive (CISSMP) ${ }^{4}$ pour effectuer un suivi du bilan de la SMP et du bienêtre au Canada. Le CISSMP est fondé sur un modèle socioécologique qui tient compte des multiples facettes d'un système social2,3. Notre aperçu intègre les dernières estimations des résultats en matière de SMP et de facteurs de risque et de protection connexes aux niveaux individuel, familial, communautaire et social pour les adultes de 18 ans et plus.

\section{Méthodologie}

Nous avons exploré les associations entre divers facteurs sociodémographiques et les résultats en matière de SMP à l'aide des données de l'Enquête sur la santé dans les collectivités canadiennes (ESCC) de 2015 et de 2017. Pour tenir compte de la conception complexe de l'ESCC, nous avons pondéré les estimations en fonction des poids d'échantillonnage fournis par Statistique Canada et de la variance estimée à l'aide de la méthode bootstrap. Nous avons réalisé cinq modèles de régression logistique ajustés. Toutes les analyses statistiques ont été réalisées à l'aide de la version 7.1 du logiciel SAS Enterprise Guide (SAS Institute Inc., Cary, Caroline du Nord, États-Unis).

Le CISSMP comprend cinq résultats en matière de SMP : santé mentale autoévaluée,

\section{Points saillants}

- Le tableau Statistiques rapides présente les estimations les plus récentes en matière de santé mentale positive et de facteurs de risque et de protection connexes chez les adultes au Canada.

- La majorité des adultes au Canada ont une santé mentale positive élevée.

- Parmi les cinq résultats en matière de santé mentale positive, la satisfaction à l'égard de la vie a la prévalence la plus élevée $(87,1 \%)$, et le sentiment de bien-être social la prévalence la plus faible $(68,1 \%)$.

- Plusieurs facteurs sociodémographiques, à savoir le sexe, le groupe d'âge, le quintile de revenu, le niveau de scolarité, la province, le milieu de résidence (urbain/rural) et le statut vis-à-vis de l'immigration sont associés de manière statistiquement significative aux résultats en matière de santé mentale positive.

bonheur, satisfaction à l'égard de la vie, bien-être psychologique et bien-être social.

\section{Santé mentale autoévaluée}

La santé mentale autoévaluée est mesurée à l'aide des données de la composante annuelle de l'ESCC de 2017. On a demandé aux répondants : "En général, diriez-vous que votre santé mentale est...?» Les choix de réponse étaient : " excellente ", " très bonne ", «bonne ", " passable » et « mauvaise ». Dans notre étude, un niveau élevé en matière de santé mentale se définit par le fait de déclarer avoir une santé mentale « excellente » ou « très bonne ». 


\section{Bonheur}

Le bonheur est mesuré à l'aide des données de la composante annuelle de l'ESCC de 2015. On a demandé aux répondants : « $\mathrm{Au}$ cours du dernier mois, à quelle fréquence vous êtes-vous senti heureux? » Les choix de réponse étaient " tous les jours ", " presque tous les jours ", " environ deux ou trois fois par semaine ", " environ une fois par semaine ", " une fois ou deux » ou " jamais ». Un niveau élevé de bonheur est défini ici par le fait de déclarer s'être senti heureux " tous les jours » ou " presque tous les jours » au cours du dernier mois.

\section{Satisfaction à l'égard de la vie}

La satisfaction à l'égard de la vie est mesurée à l'aide des données de la composante annuelle de l'ESCC de 2015. On a demandé aux répondants : "Au cours du dernier mois, à quelle fréquence vous êtes-vous senti satisfait à l'égard de votre vie? » Les choix de réponse étaient : " tous les jours ", " presque tous les jours ", " environ deux ou trois fois par semaine", " environ une fois par semaine ", " une fois ou deux » ou " jamais ». Un niveau élevé de satisfaction à l'égard de la vie est défini ici par le fait de déclarer s'être senti satisfait à l'égard de la vie " tous les jours » ou " presque tous les jours » au cours du dernier mois.

\section{Bien-être psychologique}

Le bien-être psychologique est mesuré à l'aide de six questions de l'ESCC de 2015. On a demandé aux répondants : " $\mathrm{Au}$ cours du dernier mois, à quelle fréquence avez-vous senti : 1) que vous aimiez la plupart des facettes de votre personnalité, 2) que vous viviez des expériences qui vous poussent à grandir et à devenir une meilleure personne, 3) que votre vie a un but ou une signification, 4) que vous étiez bon pour gérer les responsabilités de votre quotidien, 5) que vous étiez capable de penser ou d'exprimer vos propres idées et opinions et 6) que vous aviez des relations chaleureuses et fondées sur la confiance avec d'autres personnes? ». Les choix de réponse étaient : " tous les jours ", " presque tous les jours », " environ deux ou trois fois par semaine ", " environ une fois par semaine ", " une fois ou deux » ou " jamais ». Ces choix de réponse étaient ensuite convertis en nombre de jours : " 28 jours », « 20 jours », " 10 jours »,
« 4 jours », « 1,5 jour » et « 0 jour ». Nous avons additionné le nombre de jours obtenu en réponse aux six questions. Un bien-être psychologique élevé est défini ici par un total de 20 jours ou plus au cours du dernier mois.

\section{Bien-être social}

Le bien-être social est mesuré à l'aide des données de la composante annuelle de l'ESCC de 2017. On a demandé aux répondants : "Comment décririez-vous votre sentiment d'appartenance à votre communauté locale? Diriez-vous qu'il est...? » Les choix de réponse étaient : " très fort ", " plutôt fort ", " plutôt faible » ou " très faible ». Un sentiment de bien-être social élevé est défini ici par le fait de déclarer avoir un sentiment d'appartenance " très fort » ou " plutôt fort ».

\section{Résultats}

\section{Principales constatations}

Le tableau 1 présente l'édition 2019 des Statistiques rapides du CISSMP. Parmi les adultes de 18 ans et plus au Canada, $69,9 \%$ ont déclaré avoir un niveau élevé de santé mentale, $85,9 \%$ ont déclaré avoir un niveau élevé de bonheur, 87,1 \% ont déclaré avoir un niveau élevé de satisfaction à l'égard de la vie, 75,2 \% ont déclaré avoir un bien-être psychologique élevé et $68,1 \%$ ont déclaré avoir un sentiment de bien-être social élevé. Il ne faut pas comparer ces estimations à celles de l'édition 2016 des Statistiques rapides du CISSMP $^{4}$ car, à compter de l'édition 2015, la composante annuelle de l'ESCC a subi des changements méthodologiques importants qui ont une incidence sur les données ${ }^{5}$.

\section{Déterminants sociodémographiques et résultats en matière de SMP}

Le tableau 2 présente les rapports de cotes (RC) des résultats en matière de SMP, ajustés en fonction du sexe, de l'âge, du revenu du ménage, de la scolarité, de la province, du milieu de résidence (urbain/rural) et du statut vis-à-vis de l'immigration.

\section{Sexe}

Comparativement à l'ensemble des résultats en matière de SMP, le sexe n'était associé de façon statistiquement significative qu'à la santé mentale autodéclarée. La probabilité de déclarer une bonne santé mentale était de $18 \%$ plus élevée chez les hommes que chez les femmes (rapport de cotes ajusté $[\mathrm{RCa}]=1,18$, intervalle de confiance [IC] à $95 \%=1,11$ à 1,26).

\section{Âge}

Dans l'ensemble, les personnes de 65 ans et plus étaient proportionnellement plus nombreuses à déclarer une bonne santé mentale que celles de 18 à 64 ans. Elles étaient plus susceptibles d'avoir un niveau élevé de santé mentale autoévaluée, de bonheur, de satisfaction à l'égard de la vie, de bien-être psychologique et de bienêtre social (tableau 2).

\section{Revenu}

La probabilité d'avoir un niveau élevé de santé mentale autoévaluée, de bonheur, de satisfaction à l'égard de la vie, de bienêtre psychologique et de bien-être social augmentait de façon graduelle et statistiquement significative en fonction de la suffisance du revenu du ménage. Par exemple, les adultes du groupe au revenu le plus élevé (Q5) étaient 3,07 fois plus susceptibles d'avoir un niveau élevé de satisfaction à l'égard de la vie que les adultes du groupe au revenu le plus faible (Q1) (RCa = 3,07, IC à $95 \%=2,60$ à 3,63 ). D'autres rapports de cotes portant sur d'autres groupes de revenu sont présentés dans le tableau 2.

\section{Niveau de scolarité}

Dans l'ensemble, les diplômés postsecondaires étaient plus susceptibles de déclarer un niveau élevé de santé mentale autoévaluée, de bonheur et de satisfaction à l'égard de la vie comparativement aux diplômés du secondaire et aux personnes n’ayant pas terminé leurs études secondaires. Par exemple, les diplômés postsecondaires avaient $75 \%$ plus de chances d'avoir un niveau élevé de santé mentale autoévaluée que les personnes n'ayant pas terminé leurs études secondaires $(\mathrm{RCa}=1,75$, IC à $95 \%=1,57$ à 1,95$)$. Dans le même ordre d'idées, les chances des diplômés du secondaire d'avoir une santé mentale autoévaluée positive étaient de $35 \%$ supérieures à celles des personnes n'ayant pas obtenu leur diplôme d'études secondaires ( $\mathrm{RCa}=1,35$, IC à $95 \%=1,19$ à 1,53). Aucun lien n'a été observé entre le niveau de scolarité et le bien-être psychologique ou social. D’autres rapports de cotes sont présentés dans le tableau 2. 


\section{Province}

Quelques différences ont été observées entre les provinces en ce qui a trait à la probabilité d'avoir de bons résultats en matière de SMP. Les adultes de la Colombie-Britannique, de l'Alberta, de la Saskatchewan, de l'Ontario, du Québec, du Nouveau-Brunswick et de l'Île-duPrince-Édouard étaient proportionnellement plus nombreux à avoir un niveau élevé de bonheur que ceux de NouvelleÉcosse (tableau 2). De même, les adultes de la Saskatchewan ( $\mathrm{RCa}=1,43$, IC à $95 \%=1,03$ à 1,97), du Québec $(\mathrm{RCa}=1,57, \mathrm{IC}$ à $95 \%=1,25$ à 1,97$)$ et de l'île-du-Prince-Édouard (RCa $=1,68$, IC à $95 \%=1,17$ à 2,40 ) avaient nettement plus de chances de faire état d'une grande satisfaction à l'égard de la vie que ceux de Nouvelle-Écosse. Les adultes de Terre-Neuve-et-Labrador étaient les plus susceptibles de déclarer un niveau élevé de santé mentale autoévaluée ( $\mathrm{RCa}=1,22$, IC à $95 \%=1,01$ à 1,48) et de bien-être social $(\mathrm{RCa}=1,50, \mathrm{IC}$ à $95 \%=1,20$ à $1,87)$. D'autres rapports de cotes sont présentés dans le tableau 2.

\section{Milieu de résidence (urbain/rural)}

Le milieu de résidence (urbain/rural) était fortement associé au bonheur, à la satisfaction à l'égard de la vie et au bien-être psychologique et social. Les personnes vivant en milieu rural étaient plus susceptibles de faire état de niveaux élevés de bonheur $(\mathrm{RCa}=1,26$, IC à $95 \%=1,14$ à $1,40)$, de satisfaction à l'égard de la vie $(\mathrm{RCa}=1,26$, IC à $95 \%=1,12$ à 1,41$)$, de bien-être psychologique $(\mathrm{RCa}=1,10$, IC à $95 \%=1,01$ à 1,20) et de bien-être social $(\mathrm{RCa}=1,11$, IC à $95 \%=1,03$ à $1,19)$ que celles habitant dans les régions urbaines. Aucun lien n'a été observé entre le milieu de résidence et la santé mentale autoévaluée.

\section{Immigrants}

Les immigrants étaient proportionnellement plus nombreux que les non-immigrants à présenter un niveau élevé de santé mentale autoévaluée (RCa $=1,39$, IC à $95 \%=1,27$ à 1,51$)$ et de bien-être social $(\mathrm{RCa}=1,34$, IC à $95 \%=1,22$ à 1,48$)$. Toutefois, les immigrants étaient moins susceptibles de faire état d'un niveau de bonheur élevé ( $\mathrm{RCa}=0,76$, IC à $95 \%=$ 0,66 à 0,86 ). Aucun lien n'a été observé entre le statut vis-à-vis de l'immigration et un niveau élevé de satisfaction à l'égard de la vie ou de bien-être psychologique.

\section{Conclusion}

Cet article présente les estimations de prévalence chez les adultes tirées de l'édition 2019 du CISSMP. Nos résultats indiquent une association significative entre un âge avancé ainsi que le quintile de revenu le plus élevé et les cinq résultats positifs en matière de santé mentale. Les adultes ayant fait des études postsecondaires et ceux vivant en milieu rural étaient plus susceptibles d'éprouver du bonheur, de la satisfaction à l'égard de la vie, un bien-être psychologique et un sentiment de bien-être social. Les immigrants et les hommes avaient davantage tendance à avoir un niveau élevé de santé mentale autoévaluée que les non-immigrants et les femmes. Relever ces différences sociodémographiques dans les résultats en matière de SMP contribue à une meilleure compréhension de la santé mentale positive des adultes.

\section{Conflits d'intérêts}

Les auteures déclarent n'avoir aucun conflit d'intérêts.

\section{Contributions des auteures et avis}

$\mathrm{MV}, \mathrm{EP}, \mathrm{TL}$ et $\mathrm{MB}$ ont rédigé cet aperçu. MV a analysé les estimations de prévalence concernant la santé mentale autoévaluée et a réalisé les analyses du modèle de régression. Toutes les coauteures ont interprété les données et examiné ou révisé l'aperçu.

Le contenu de l'article et les points de vue qui y sont exprimés n'engagent que les auteures; ils ne correspondent pas nécessairement à ceux du gouvernement du Canada.

\section{Références}

1. Agence de la santé publique du Canada. Promotion de la santé mentale : Promouvoir la santé mentale, c'est promouvoir le meilleur de nous-mêmes [Internet]. Ottawa (Ont.) : Agence de la santé publique du Canada; [modifié le 6 mai 2014; consultation le 25 juin 2019]. En ligne à : https://www.canada .ca/fr/sante-publique/services/promotion -sante/sante-mentale/promotion-sante -mentale.html
2. Orpana H, J Vachon, J Dykxhoorn, et al. Surveillance de la santé mentale positive et de ses facteurs déterminants au Canada : élaboration d'un cadre d'indicateurs de surveillance de la santé mentale positive. Promotion de la santé et prévention des maladies chroniques au Canada. 2016; 36(1):1-12.

3. Agence de la santé publique du Canada (ASPC). Plan ministériel, 2019-2020. [Internet]. Ottawa (Ont.) :ASPC; 2019 [consultation le 21 mai 2019]. En ligne à : https://www.canada.ca/content /dam/phac-aspc/documents/corporate /transparency/corporate-management -reporting/reports-plans-priorities /2019-2020-report-plans-priorities /phac-aspc-2019-2020-departmental -plan-fra.pdf

4. Centre de prévention des maladies chroniques. Cadre d'indicateurs pour la surveillance de la santé mentale positive - Statistiques rapides, adultes (18 ans et plus), Canada, édition 2016. Promotion de la santé et prévention des maladies chroniques au Canada. 2016;36(1):12-13.

5. Statistique Canada. Enquête sur la santé dans les collectivités canadiennes - Composante annuelle (ESCC) [Internet]. Ottawa (Ont.) : Statistique Canada; 2018 [consultation le 3 juillet 2019]. En ligne à : http://www23 .statcan.gc.ca/imdb/p2SV_f.pl ?Function $=$ getSurvey $\& I d=329241$ 


\section{TABLEAU 1}

\section{CADRE D'INDICATEURS DE SURVEILLANCE DE LA SANTÉ MENTALE POSITIVE STATISTIQUES RAPIDES, ADULTES (18 ANS ET PLUS), CANADA, ÉDITION 2019}

\begin{tabular}{|c|c|c|c|}
\hline GROUPE D'INDICATEURS & MESURE(S) LIÉE(S) À L'INDICATEUR & $\begin{array}{l}\text { DONNÉES } \\
\text { LES PLUS } \\
\text { RÉCENTES }\end{array}$ & $\begin{array}{l}\text { SOURCE DE } \\
\text { DONNÉES } \\
\text { (ANNÉE) }\end{array}$ \\
\hline \multicolumn{4}{|c|}{ RÉSULTATS EN MATIÈRE DE SANTÉ MENTALE POSITIVE } \\
\hline Santé mentale autoévaluée & $\%$ de la population ayant déclaré avoir une « excellente » ou une «très bonne » santé mentale & $69,9 \%$ & ESCC (2017) \\
\hline Bonheur & \% de la population ayant déclaré être heureuse «tous les jours» ou « presque tous les jours» & $85,9 \%$ & ESCC (2015) \\
\hline \multirow[t]{2}{*}{$\begin{array}{l}\text { Satisfaction à l'égard de la } \\
\text { vie }\end{array}$} & $\begin{array}{l}\% \text { de la population ayant déclaré être satisfaite à l'égard de la vie « tous les jours » ou « presque } \\
\text { tous les jours» }\end{array}$ & $87,1 \%$ & ESCC (2015) \\
\hline & Moyenne de l'estimation de satisfaction à l'égard de la vie (échelle de 0 à 10) & 8,1 & ESCC (2017) \\
\hline Bien-être psychologique & \% de la population ayant un niveau élevé de bien-être psychologique & $75,2 \%$ & ESCC (2015) \\
\hline Bien-être social & $\begin{array}{l}\text { \% de la population ayant fait état d'un sentiment d'appartenance « très fort » ou « plutôt fort » à } \\
\text { sa communauté locale }\end{array}$ & $68,1 \%$ & ESCC (2017) \\
\hline \multicolumn{4}{|c|}{ DÉTERMINANTS INDIVIDUELS } \\
\hline Résilience & \multicolumn{3}{|l|}{ En cours d'élaboration } \\
\hline Capacité d'adaptation & \% de la population ayant déclaré avoir une forte capacité d'adaptation & $56,9 \%$ & $\begin{array}{l}\text { ESCC - Santé } \\
\text { mentale (2012) }\end{array}$ \\
\hline Contrôle et autoefficacité & \% de la population ayant déclaré un niveau élevé de maîtrise de sa destinée & $41,6 \%$ & $\begin{array}{l}\text { ESG - Réseaux } \\
\text { sociaux (2008) }\end{array}$ \\
\hline \multirow[t]{3}{*}{ Violence } & $\begin{array}{l}\text { \% de la population ayant déclaré avoir été victime, avant l'âge de } 15 \text { ans, de l'un ou l'autre de } \\
\text { ces trois types de violence durant l'enfance : violence physique ou sexuelle de la part d'un } \\
\text { adulte, et/ou exposition à la violence commise par les parents ou les tuteurs }\end{array}$ & $34,0 \%$ & $\begin{array}{l}\text { ESG - Victimisation } \\
\text { (2014) }\end{array}$ \\
\hline & $\begin{array}{l}\text { \% de la population ayant déclaré avoir été victime de violence physique ou sexuelle au cours des } \\
12 \text { derniers mois }\end{array}$ & $3,9 \%$ & $\begin{array}{l}\text { ESG - Victimisation } \\
\text { (2014) }\end{array}$ \\
\hline & $\begin{array}{l}\text { \% de la population ayant déclaré avoir été victime de violence conjugale au cours des cinq } \\
\text { dernières années }\end{array}$ & $2,7 \%$ & $\begin{array}{l}\text { ESG - Victimisation } \\
\text { (2014) }\end{array}$ \\
\hline \multirow[t]{2}{*}{ État de santé } & \% de la population ayant déclaré avoir une « excellente » ou « très bonne » santé & $59,9 \%$ & ESCC (2017) \\
\hline & \% de la population n’ayant aucune incapacité ou ayant une incapacité légère & $68,5 \%$ & ESCC (2015) \\
\hline \multirow[t]{2}{*}{ Activité physique } & $\begin{array}{l}\% \text { de la population « active » ou « modérément active » durant ses loisirs, selon des données } \\
\text { autodéclarées }\end{array}$ & $69,4 \%$ & ESCC (2017) \\
\hline & $\begin{array}{l}\text { \% des personnes de } 18 \text { à } 79 \text { ans qui suivent les directives en matière d'activité physique en } \\
\text { pratiquant au moins } 150 \text { minutes d'activité physique d'intensité modérée à élevée chaque } \\
\text { semaine, en séances de } 10 \text { minutes ou plus }\end{array}$ & $16,4 \%$ & $\begin{array}{c}\text { ECMS } \\
(2016-2017)\end{array}$ \\
\hline $\begin{array}{l}\text { Consommation de } \\
\text { substances }\end{array}$ & $\begin{array}{l}\text { \% de la population dont la consommation déclarée d'alcool respecte les lignes directrices sur la } \\
\text { consommation d'alcool à faible risque }\end{array}$ & $83,4 \%$ & ECTAD (2017) \\
\hline Spiritualité & $\begin{array}{l}\text { \% de la population ayant déclaré que les croyances religieuses ou spirituelles sont « très } \\
\text { importantes » ou « importantes » dans leur vie quotidienne }\end{array}$ & $62,7 \%$ & $\begin{array}{l}\text { ESCC - Santé } \\
\text { mentale (2012) }\end{array}$ \\
\hline \multicolumn{4}{|l|}{ DÉTERMINANTS FAMILIAUX } \\
\hline Relations familiales & \multicolumn{3}{|l|}{ En cours d'élaboration } \\
\hline \multirow{2}{*}{$\begin{array}{l}\text { État de santé de la famille } \\
\text { et consommation de } \\
\text { substances des membres de } \\
\text { la famille }\end{array}$} & $\begin{array}{l}\text { \% de la population ayant au moins un membre de leur famille qui a des problèmes concernant } \\
\text { ses émotions, sa santé mentale ou sa consommation d'alcool ou de drogues }\end{array}$ & $39,8 \%$ & $\begin{array}{l}\text { ESCC - Santé } \\
\text { mentale (2012) }\end{array}$ \\
\hline & $\begin{array}{l}\text { \% de la population ayant au moins un membre de leur famille qui a des problèmes concernant } \\
\text { ses émotions, sa santé mentale ou sa consommation d'alcool ou de drogues et déclarant que les } \\
\text { problèmes de ce membre de la famille affectent « beaucoup » ou « assez » leur vie }\end{array}$ & $35,6 \%$ & $\begin{array}{l}\text { ESCC - Santé } \\
\text { mentale (2012) }\end{array}$ \\
\hline \multirow[t]{3}{*}{ Composition du ménage } & $\%$ de la population vivant avec un conjoint ou un partenaire & $70,5 \%$ & ESCC (2017) \\
\hline & $\%$ de la population vivant dans un ménage monoparental & $8,8 \%$ & ESCC (2017) \\
\hline & $\%$ de la population vivant seule & $16,1 \%$ & ESCC (2017) \\
\hline Revenu du ménage & $\begin{array}{l}\text { \% de la population canadienne totale, de tous les âges, vivant sous le seuil de faible revenu après } \\
\text { impôt }\end{array}$ & $8,8 \%$ & EDTR (2011) \\
\hline
\end{tabular}


DÉTERMINANTS COMMUNAUTAIRES

\begin{tabular}{|c|c|c|c|}
\hline Implication communautaire & $\begin{array}{l}\text { \% de la population membre de (ou participant à) au moins un organisme, un groupe, une } \\
\text { association ou un club récréatif ou professionnel }\end{array}$ & $63,4 \%$ & $\begin{array}{l}\text { ESG - Identité } \\
\text { sociale (2013) }\end{array}$ \\
\hline \multirow[t]{2}{*}{ Réseaux sociaux } & $\begin{array}{l}\% \text { de la population ayant déclaré n'avoir aucun ami ou membre de la famille dont elle soit } \\
\text { proche }\end{array}$ & $6,1 \%$ & $\begin{array}{l}\text { ESG - Identité } \\
\text { sociale (2013) }\end{array}$ \\
\hline & $\begin{array}{l}\text { \% de la population ayant déclaré avoir au moins } 6 \text { amis ou membres de la famille dont elle soit } \\
\text { proche }\end{array}$ & $36,8 \%$ & $\begin{array}{l}\text { ESG - Identité } \\
\text { sociale (2013) }\end{array}$ \\
\hline Environnement de travail & \% des salariés de 18 à 75 ans vivant un niveau de stress élevé au travail & $14,8 \%$ & $\begin{array}{l}\text { ESCC - Santé } \\
\text { mentale (2012) }\end{array}$ \\
\hline \multirow[t]{2}{*}{$\begin{array}{l}\text { Environnement social du } \\
\text { quartier }\end{array}$} & \% de la population ayant déclaré vivre dans un quartier où les gens s'entraident & $88,5 \%$ & $\begin{array}{l}\text { ESG - Victimisation } \\
\text { (2014) }\end{array}$ \\
\hline & $\begin{array}{l}\text { \% de la population ayant déclaré que le désordre social dans leur quartier est un « très gros } \\
\text { problème » ou un « assez gros problème » }\end{array}$ & $5,3 \%$ & $\begin{array}{l}\text { ESG - Victimisation } \\
\text { (2014) }\end{array}$ \\
\hline \multicolumn{4}{|l|}{ DÉTERMINANTS SOCIAUX } \\
\hline Inégalité & \multicolumn{3}{|l|}{ En cours d'élaboration } \\
\hline \multirow[t]{2}{*}{$\begin{array}{l}\text { Discrimination et } \\
\text { stigmatisation }\end{array}$} & $\begin{array}{l}\text { \% de la population ayant subi un traitement injuste au moins une fois au cours des cinq années } \\
\text { précédentes en raison, entre autres, de leur sexe, race, âge ou apparence }\end{array}$ & $11,5 \%$ & $\begin{array}{l}\text { ESG - Victimisation } \\
\text { (2014) }\end{array}$ \\
\hline & $\begin{array}{l}\text { \% de la population atteinte d'un problème de santé mentale déclarant avoir été affectée par des } \\
\text { opinions négatives ou un traitement injuste en raison de ce problème }\end{array}$ & $21,0 \%$ & $\begin{array}{c}\text { ESCC - Santé } \\
\text { mentale (2012) }\end{array}$ \\
\hline Participation politique & \% des électeurs inscrits ayant voté aux élections fédérales de 2015 & $68,3 \%$ & $\begin{array}{c}\text { Élections } \\
\text { Canada (2015) }\end{array}$ \\
\hline
\end{tabular}

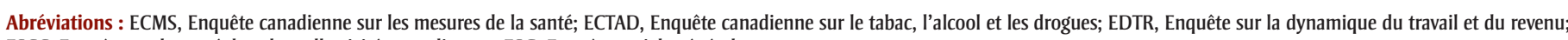
ESCC, Enquête sur la santé dans les collectivités canadiennes; ESG, Enquête sociale générale.

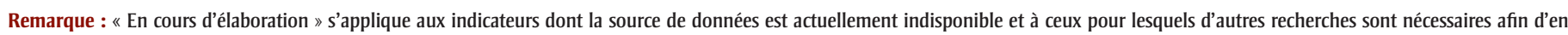
déterminer une mesure et une source de données satisfaisantes.

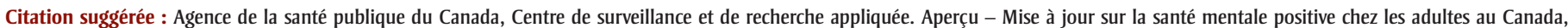
Statistiques rapides, adultes (18 ans et plus), Canada, édition 2019. Ottawa (Ont.) : Agence de la santé publique du Canada; 2020.

Si vous avez des questions ou des commentaires, veuillez communiquer avec nous à l'adresse suivante : phac.infobase.aspc@canada.ca 
TABLEAU 2

Rapports de cotes ajustés des résultats en matière de santé mentale positive, adultes (18 ans et plus), Canada, 2015 et 2017

\begin{tabular}{|c|c|c|c|c|c|}
\hline \multirow{2}{*}{ Variable } & SMAE & Bonheur ${ }^{b}$ & $\begin{array}{c}\text { Satisfaction à l'égard } \\
\text { de la vie } \text { vi }^{\text {b }}\end{array}$ & $\begin{array}{c}\text { Bien-être } \\
\text { psychologique }^{\text {b }}\end{array}$ & Bien-être sociala \\
\hline & RCa (IC à 95 \%) & RCa (IC à 95 \%) & RCa (IC à $95 \%$ ) & RCa (IC à 95 \%) & RCa (IC à $95 \%$ ) \\
\hline \multicolumn{6}{|l|}{ Sexe } \\
\hline Femmes & Réf. & Réf. & & & Réf. \\
\hline Hommes & 1,18 (1,11 à 1,26) & $0,95(0,86$ à 1,04$)$ & 0,98 (0,89 à 1,09) & 1,05 (0,98 à 1,14) & $0,95(0,89$ à 1,02$)$ \\
\hline \multicolumn{6}{|l|}{ Âge } \\
\hline 18 à 24 ans & $0,85(0,74$ à 0,97$)$ & $1,40(1,16$ à 1,70$)$ & 1,07 (0,89 à 1,28) & $0,84(0,73$ à 0,96$)$ & $0,94(0,82$ à 1,07$)$ \\
\hline 25 à 44 ans & $1,00(0,92$ à 1,09$)$ & 1,31 (1,16 à 1,49) & 1,04 (0,91 à 1,18) & 0,97 (0,88 à 1,08) & $0,87(0,80$ à 0,94$)$ \\
\hline 45 à 64 ans & Réf. & Réf. & Réf. & Réf. & Réf. \\
\hline 65 ans + & 1,32 (1,22 à 1,44) & 1,61 (1,42 à 1,83) & 1,88 (1,62 à 2,18) & 1,34 (1,20 à 1,50) & 1,67 (1,54 à 1,82) \\
\hline \multicolumn{6}{|l|}{ Suffisance du revenu du ménage } \\
\hline Q1 (faible) & Réf. & Réf. & Réf. & Réf. & Réf. \\
\hline Q2 & $1,29(1,17$ à 1,42$)$ & 1,34 (1,15 à 1,56) & 1,30 (1,11 à 1,52) & $1,04(0,91$ à 1,18) & $1,13(1,01$ à 1,25$)$ \\
\hline Q3 & $1,56(1,41$ à 1,72$)$ & 1,96 (1,68 à 2,28) & 2,09 (1,78 à 2,45) & $1,17(1,03$ à 1,32) & $1,29(1,15$ à 1,43$)$ \\
\hline Q4 & $1,81(1,62$ à 2,01$)$ & 2,19 (1,88 à 2,55) & 2,34 (1,98 à 2,77) & $1,42(1,25$ à 1,61$)$ & $1,28(1,15$ à 1,42$)$ \\
\hline Q5 (élevée) & 2,28 (2,05 à 2,54) & $2,67(2,29$ à 3,11$)$ & $3,07(2,60$ à 3,63$)$ & $1,60(1,40$ à 1,82) & $1,31(1,17$ à 1,46$)$ \\
\hline \multicolumn{6}{|l|}{ Niveau de scolarité le plus élevé (ménage) } \\
\hline Études secondaires non terminées & Réf. & Réf. & Réf. & Réf. & Réf. \\
\hline Diplôme d'études secondaires & 1,35 (1,19 à 1,53) & 1,23 (1,03 à 1,47) & $0,96(0,81$ à 1,14$)$ & 1,03 (0,89 à 1,21) & $0,96(0,83,1,10)$ \\
\hline $\begin{array}{l}\text { Diplôme d'études postsecon- } \\
\text { daires }\end{array}$ & 1,75 (1,57 à 1,95) & 1,37 (1,16 à 1,63) & 1,24 (1,06 à 1,46) & 1,11 (0,96 à 1,28) & $1,09(0,97$ à 1,23$)$ \\
\hline \multicolumn{6}{|l|}{ Province } \\
\hline Colombie-Britannique & $0,96(0,83$ à 1,11$)$ & 1,34 (1,08 à 1,65) & $0,96(0,75$ à 1,22$)$ & 0,93 (0,76 à 1,13) & $1,02(0,87$ à 1,20$)$ \\
\hline Alberta & $1,00(0,87$ à 1,16$)$ & 1,26 (1,02 à 1,56) & 1,07 (0,85 à 1,34) & 1,02 (0,85 à 1,24) & $0,92(0,78$ à 1,08$)$ \\
\hline Saskatchewan & $0,97(0,81$ à 1,17$)$ & 1,41 (1,07 à 1,87) & 1,43 (1,03 à 1,97) & 0,96 (0,77 à 1,20) & $1,21(0,99$ à 1,48$)$ \\
\hline Manitoba & $1,09(0,91$ à 1,31$)$ & $1,25(0,98$ à 1,61) & $1,23(0,91$ à 1,65$)$ & $1,02(0,82$ à 1,27$)$ & $1,13(0,94$ à 1,36$)$ \\
\hline Ontario & $1,04(0,91$ à 1,18$)$ & 1,29 (1,06 à 1,58) & 1,19 (0,95 à 1,49) & 0,95 (0,79 à 1,14) & $1,04(0,89$ à 1,21$)$ \\
\hline Québec & 1,42 (1,24 à 1,62) & 1,37 (1,12 à 1,68) & 1,57 (1,25 à 1,97) & $0,89(0,75$ à 1,07$)$ & $0,69(0,59$ à 0,80$)$ \\
\hline Nouveau-Brunswick & $1,03(0,85$ à 1,24$)$ & 1,44 (1,08 à 1,92) & 1,24 (0,92 à 1,68) & 1,08 (0,86 à 1,37) & $1,37(1,10$ à 1,70$)$ \\
\hline Nouvelle-Écosse & Réf. & Réf. & Réf. & Réf. & Réf. \\
\hline île-du-Prince-Édouard & $1,05(0,85$ à 1,31$)$ & 1,68 (1,20 à 2,36) & 1,68 (1,17 à 2,40) & 1,59 (1,21 à 2,09) & 1,34 (1,06 à 1,70) \\
\hline Terre-Neuve-et-Labrador & 1,22 (1,01 à 1,48) & 1,18 (0,91 à 1,54) & 1,27 (0,94 à 1,71) & 1,41 (1,11 à 1,78) & $1,50(1,20$ à 1,87$)$ \\
\hline \multicolumn{6}{|l|}{ Milieu de résidence } \\
\hline Rural & $1,01(0,94$ à 1,08$)$ & 1,26 (1,14 à 1,40) & $1,26(1,12$ à 1,41) & $1,10(1,01$ à 1,20) & $1,11(1,03$ à 1,19$)$ \\
\hline Urbain & Réf. & Réf. & Réf. & Réf. & Réf. \\
\hline \multicolumn{6}{|l|}{ Immigrant } \\
\hline Oui & $1,39(1,27$ à 1,51) & 0,76 (0,66 à 0,86) & 0,91 (0,79 à 1,06) & 1,02 (0,91 à 1,15) & 1,34 (1,22 à 1,48) \\
\hline Non & Réf. & Réf. & Réf. & Réf. & Réf. \\
\hline
\end{tabular}

Abréviations : IC, intervalle de confiance; Q, quintile; RCa, rapport de cotes ajusté; Réf., groupe de référence; SMAE, santé mentale autoévaluée.

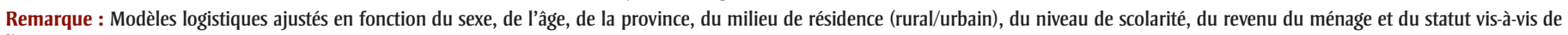
l'immigration.

a Toutes ces estimations sont tirées des données de l'ESCC de 2017.

b Toutes ces estimations sont tirées des données de l'ESCC de 2015. 